\title{
Avaliação da qualidade físico química de alisantes capilares
}

\author{
Quality of the chemical substance of hair straighteners \\ Evaluación de la calidad físico-química de las planchas para el cabello
}

Recebido: 05/10/2021 | Revisado: 13/10/2021 | Aceito: 21/10/2021 | Publicado: 23/10/2021

\author{
Anny Louisy de Sousa Macêdo \\ ORCID: https://orcid.org/0000-0003-3924-3766 \\ Centro Universitário UNIFACID, Brasil \\ E-mail: annylouisy@hotmail.com \\ Cristian José Oliveira \\ ORCID: https://orcid.org/0000-0003-4036-1604 \\ Centro Universitário UNIFACID, Brasil \\ E-mail: cristianoliveira59@hotmail.com \\ Diego da Trindade Ribeiro \\ ORCID: https://orcid.org/0000-0001-7261-8369 \\ Centro Universitário UNIFACID, Brasil \\ E-mail: diegojurema@ hotmail.com \\ Viviane Ferreira de Sousa \\ ORCID: https://orcid.org/0000-0001-8049-8213 \\ Centro Universitário UNIFACID, Brasil \\ E-mail: vivi.fs@outlook.com \\ Mayara Ladeira Coêlho \\ ORCID: https://orcid.org/0000-0002-8084-5964 \\ Centro Universitário UNIFACID, Brasil \\ E-mail: mayara.coelho@ facid.edu.br
}

\begin{abstract}
Resumo
A pesquisa teve como objetivo avaliar a qualidade físico-química de produtos utilizados como alisantes capilares. Os parâmetros utilizados foram medidas de $\mathrm{pH}$, viscosidade, características organolépticas, centrifugação, espalhabilidade e busca por substâncias proibitivas. Todos os testes foram realizados com embasamento no Guia de Controle de Qualidade de Produtos Cosméticos, publicado pela Agência Nacional de Vigilância Sanitária (ANVISA). Foram avaliadas 10 amostras de alisantes, as quais foram identificadas por meio de letras a fim de manter o sigilo. O método de pesquisa realizado foi do tipo experimental com abordagem quantitativa. Os testes revelaram que as 10 amostras apresentaram valor de $\mathrm{pH}$ fora das especificações $(5,0$ a 7,0), quanto a viscosidade foi possível identificar que a amostra com maior viscosidade foi a do produto A5 (6486 cP) ao passo que a amostra com menor viscosidade foi a A8 $(801 \mathrm{cP})$. Na busca de substâncias proibitivas, todas as amostras foram positivas no teste para detecção de formaldeído com presença acima de 0,01\% de formol. Sendo assim, faz-se necessário um controle mais rígido para alisantes capilares tendo em vista a inadequação de alguns parâmetros avaliados.
\end{abstract}

Palavras-chave: Controle de qualidade; Rotulagem de produtos; Indústria cosmética; Formaldeído.

\begin{abstract}
The research aimed to evaluate the physicochemical quality, and labeling of products used as hair straighteners. The parameters used were measurements of $\mathrm{pH}$, viscosity, organoleptic characteristics, centrifugation, spreadability and prohibitive substance search. All tests were performed based on the Quality Control Guide for Cosmetic Products, published by the National Health Surveillance Agency (ANVISA). Ten samples of straighteners were evaluated, which were identified by letters in order to maintain confidentiality. The research method was experimental type with quantitative approach. Data analysis showed that the 10 samples had a $\mathrm{pH}$ value outside the specifications (5.0 to 7.0). Regarding the viscosity of the straighteners, it was possible to observe that the sample with the highest viscosity was the product sample A5 $(6486 \mathrm{cP})$ whereas the sample with the lowest viscosity was sample A8 $(801 \mathrm{cP})$. In the search for prohibitive substances, all samples were positive in the test for detecting formaldehyde with presence above $0,01 \%$ of formalin. Therefore, a stricter control for hair straighteners is necessary in view of the inadequacy of some evaluated parameters.
\end{abstract}

Keywords: Quality control; Product labeling; Cosmetic industry; Formaldehyde.

\section{Resumen}

La investigación tuvo como objetivo evaluar la calidad fisicoquímica de los productos utilizados como alisadores de cabello. Los parámetros utilizados fueron medidas de $\mathrm{pH}$, viscosidad, características organolépticas, centrifugación, esparcimiento y búsqueda de sustancias prohibitivas. Todas las pruebas se realizaron con base en 
la Guía de Control de Calidad de Productos Cosméticos, publicada por la Agencia Nacional de Vigilancia Sanitaria (ANVISA). Se evaluaron diez muestras de alisadores, las cuales fueron identificadas por letras con el fin de mantener la confidencialidad. El método de investigación realizado fue de tipo experimental con enfoque cuantitativo. El análisis de los datos determinó que las 10 muestras tenían un valor de pH fuera de las especificaciones (5.0 a 7.0), en cuanto a la viscosidad, se pudo identificar que la muestra con mayor viscosidad fue el producto A5 $(6486 \mathrm{cP})$ en el paso que el la muestra con la viscosidad más baja fue A8 (801 cP). En la búsqueda de sustancias prohibitivas, todas las muestras resultaron positivas en la prueba de detección para formaldehído con una presencia superior al $0,01 \%$ de formaldehído. Por tanto, es necesario un control más estricto de las planchas de pelo ante la insuficiencia de algunos parámetros evaluados.

Palabras clave: Control de calidad; Etiquetado de productos; Industria cosmética; Formaldehído.

\section{Introdução}

O mercado de beleza, incluindo os produtos de higiene pessoal, perfumes e cosméticos é um dos que mais crescente dentre todos os seguimentos do mercado. Segundo dados levantados pela Associação Brasileira da Indústria de Higiene Pessoal, Perfumes e Cosméticos, o Brasil encontra-se como quarto maior mercado de venda, conforme dados do Euromonitor de 2016, com um faturamento de aproximadamente R \$ 42,6 bilhões no ano de 2016, sendo o terceiro do ranking em produtos destinados aos cabelos (Abihpec, 2016).

Os alisantes são produtos que alisam, relaxam, amaciam e reduzem o volume dos cabelos, de maneira mais ou menos duradoura. Estes produtos possuem substâncias que são irritantes para a pele e se utilizados inadequadamente, podem causar queimaduras graves no couro cabeludo, quebra dos fios e queda dos cabelos (Anvisa, 2018).

De acordo com Guia de controle de qualidade de produtos cosméticos e exigido a apresentação dos dados de Controle de Qualidade (especificações) no ato da regularização do produto, pois existem substâncias ativas específicas com propriedades alisantes como ácido tioglicólico, hidróxido de sódio, hidróxido de potássio, hidróxido de cálcio, hidróxido de lítio, hidróxido de guanidina permitidas pela legislação, substâncias como formol e glutaraldeído não são permitidos como produtos alisantes, a legislação sanitária permite o uso de formol e glutaraldeído em produtos cosméticos capilares apenas na função de conservantes (com limite máximo de $0,2 \%$ e $0,1 \%$, respectivamente), e como agente endurecedor de unhas a uma concentração de até 5\%, conforme a Resolução No15 de 26 de março de 2013 (Anvisa, 2007).

Durante a fabricação do produto a adição de formol, glutaraldeído ou qualquer outra substância a um produto acabado, pronto para uso, constitui infração sanitária, estando o estabelecimento que adota esta prática sujeito às sanções administrativas, cíveis e penais cabíveis, sendo que adulteração desses produtos configura crime hediondo (Anvisa, 2018).

Com o intuito de garantir a segurança e eficácia dos produtos cosméticos, foi criado e implantado o sistema de cosmetovigilância no Brasil, através da Resolução RDC No 332, de $1^{\circ}$ de dezembro de 2005. Essa resolução foi elaborada a partir da resolução do Mercosul MERCOSUR N 19/2005, a qual determina aos países integrantes a implantação deste sistema, sendo esta função delegada aos órgãos nacionais competentes de cada país associado (Mercosul, 2005; Brasil, 2005).

A cosmetovigilância tem a função de acompanhar a resposta que o produto provocará no mercado, analisar os eventos adversos provocados por cosméticos, identificar o risco envolvido no uso desses produtos e tomar condutas pertinentes de acordo com a relação de causa estabelecida (Brasil, 2019).

Dessa forma, o objetivo geral deste trabalho foi avaliar a qualidade físico-química, e rotulagem de produtos utilizados como alisantes capilares. Especificamente objetivou-se realizar cosmetovigilância dos parâmetros compulsórios quanto às rotulagens e normativas vigentes de alisantes capilares, avaliar a qualidade físico-química de alisantes capilares quanto a determinação do pH, viscosidade, cor, odor, espalhabilidade, estabilidade e busca de substâncias proibitivas. 


\section{Metodologia}

\section{Aquisição de Amostras}

Para realização do trabalho, foram adquiridas 10 amostras de alisantes capilares de marcas diferentes e lacradas em lojas de cosméticos localizadas na cidade de Teresina-PI. As formas farmacêuticas das amostras neste estudo foram creme, nos quais submetidos a testes de avaliação de qualidade preconizados pela legislação vigente.

\section{Avaliação das características organolépticas das amostras}

Observou-se visualmente, verificando se a amostra continua com as mesmas características ou se ocorreram alterações do tipo de turvação ou separação de fazes. Analisou-se visualmente sobre condição de luz branca original. Verificou-se o odor através do olfato. Cor e Odor devem permanecer estável pôr no mínimo 15 dias a luz solar. Pequenas alterações são aceitáveis em temperaturas elevadas (Anvisa, 2008).

\section{Avaliação do pH}

Determinou-se o $\mathrm{pH}$ em triplicata através do potenciométrico digimed DM 22 em temperatura ambiente. Onde previamente calibrou-se com solução tampão pH 4,0 e 7.0, logo após as amostras foram diluídas a 10\% (p/v) em água destilada, e posteriormente fez-se a análise na diluição (Brasil, 2010).

\section{Avaliação da viscosidade}

Determinou-se a viscosidade em triplicata em um viscosímetro rotativo analógico. Utilizou-se $40 \mathrm{~g}$ de cada formulação, spindle quatro $(\mathrm{SP}=4)$ e velocidade de $6 \mathrm{rpm}$. A seguir inseriu-se o rotor verticalmente na amostra isenta de bolhas até a marca (sulco) da haste do rotor e nivelando-se o aparelho, procedeu-se à leitura da viscosidade, de acordo com o procedimento operacional do aparelho (Brasil, 2008).

\section{Avaliação da Identificação por colorimetria de Formaldeído Livre}

Preparou-se o reagente de Schiff: Pesou-se $100 \mathrm{mg}$ de fucsina em um béquer e dissolve-se em $75 \mathrm{ml}$ de água a $80^{\circ} \mathrm{C}$. Após resfriamento, acrescentou-se 2,5 g de sulfito de sódio heptaidratado e carvão ativado, completando-se até $100 \mathrm{ml}$.

Pesou-se analiticamente $2 \mathrm{~g}$ de cada amostra em um béquer de $10 \mathrm{ml}$. Juntou-se duas gotas de ácido sulfúrico $1 \mathrm{M}$ e $2 \mathrm{ml}$ de reagente de Schiff. Esse reagente estava incolor no momento da utilização. Agitou-se e deixou-se reagir durante cinco minutos. O surgimento de uma coloração rosa ou malva, após cinco minutos, indicou-se a presença de uma quantidade de formaldeído superior a $0,01 \%$ (Anvisa, 2018).

\section{Teste de estabilidade preliminar por centrifugação}

Colocou-se 5g de cada amostra em 10 tubos de ensaios cônicos para centrífuga e submetidas a ciclos de 1000 e $2500 \mathrm{rpm}$ durante 15 minutos cada. A homogeneidade das formulações em estudo foi avaliada através da observação macroscópica de separação de fases após a realização do procedimento descrito (Brasil, 2008).

\section{Avaliação da espalhabilidade}

Na determinação da espalhabilidade colocou-se uma placa molde de vidro (diâmetro $=20 \mathrm{~cm}$; espessura 0,3 ) sobre um suporte de madeira $40 \mathrm{~cm}$ por $40 \mathrm{~cm}$ ). Sob a placa posicionou-se uma folha de papel milimetrado. Introduziu-se $1 \mathrm{~g}$ da amostra no centro da placa e nivelou-se a com espátula. Sobre a amostra colocou-se uma placa de vidro de peso prédeterminado. Depois de um minuto, foi calculada a superfície abrangida, através da medição do diâmetro em duas posições 
opostas, com o auxílio da escala de papel milimetrado e com posterior cálculo do diâmetro médio. Repetiu-se este procedimento acrescentando-se novas placas, em intervalos de um minuto, registrando-se a cada determinação a superfície abrangida pela amostra e o peso da placa adicionada até um número máximo de 10 placas (Knorst, 1991).

A espalhabilidade (Ei), determinada a $27 \pm 2{ }^{\circ} \mathrm{C}$, foi calculada através da equação:

$\mathrm{Ei}=\mathrm{d} 2 \mathrm{xp} / 4$

Onde: Ei: espalhabilidade da amostra para o peso i (mm2).

D: diâmetro médio $(\mathrm{mm})$

A espalhabilidade máxima foi considerada como o ponto no qual a adição de peso não ocasionou alterações significativas nos valores da espalhabilidade, e o esforço limite corresponde ao peso que resulta na espalhabilidade máxima.

\section{Resultados e Discussão}

As características organolépticas (cor, odor, aspecto) constituem um indicativo da qualidade de uma preparação cosmética, podendo detectar possíveis alterações físico-químicas ou microbiológicas na formulação desta. O exame visual funciona como um sinalizador da homogeneidade da preparação. A cor e o odor geralmente caracterizam o estado de conservação da preparação, já que uma mudança nestes é um possível indício de alteração (Brasil, 2012).

Em relação ao aspecto e cor, as amostras apresentaram-se como cremes homogêneos, todas brancas, porém sem apresentar nenhum tipo de precipitado conforme é descrito na tabela 1. Quanto ao odor, foi possível sentir o cheiro característico intenso de enxofre nas amostras A1, A4 e A8 devido ao ácido tioglicólico, e formol nas amostras A9 e A10, no momento da observação essas amostras provocaram um pouco de irritação nas mucosas e ardência, sintomas sugestivos daqueles causados pela exposição ao formaldeído. As amostras A2, A3, A5, A6 e A7 apresentaram somente um odor agradável de essência usada nos produtos. Na amostra A9 e A10 apesar de apresentar um odor de essência característico, ainda assim era possível sentir a presença do formaldeído, ou seja, não conseguiu mascarar o odor do formol.

Tabela 1: Descrição das características organolépticas, identificação colorimétrica do formaldeído e teste de centrifugação dos alisantes capilares.

\begin{tabular}{lllllllllll}
\hline $\begin{array}{l}\text { AMOSTRAS/ } \\
\text { ENSAIOS }\end{array}$ & $\mathbf{A 1}$ & $\mathbf{A 2}$ & $\mathbf{A 3}$ & $\mathbf{A 4}$ & $\mathbf{A 5}$ & $\mathbf{A 6}$ & $\mathbf{A 7}$ & $\mathbf{A 8}$ & $\mathbf{A 9}$ & $\mathbf{A 1 0}$ \\
\hline Aspecto & $\mathrm{CH}$ & $\mathrm{CH}$ & $\mathrm{CH}$ & $\mathrm{CH}$ & $\mathrm{CH}$ & $\mathrm{CH}$ & $\mathrm{CH}$ & $\mathrm{CH}$ & $\mathrm{CH}$ & $\mathrm{CH}$ \\
Cor & $\mathrm{Branca}$ & $\mathrm{Branca}$ & $\mathrm{Branca}$ & $\mathrm{Branca}$ & $\mathrm{Branca}$ & $\mathrm{Branca}$ & Branca & Branca & Branca & Branca \\
Odor & $\mathrm{E}$ & $\mathrm{S}$ & $\mathrm{S}$ & $\mathrm{E}$ & $\mathrm{S}$ & $\mathrm{S}$ & $\mathrm{S}$ & $\mathrm{E}$ & $\mathrm{F}$ & $\mathrm{F}$ \\
$\begin{array}{l}\text { Identificação } \\
\text { colorimétrica } \\
\text { formaldeído }\end{array}$ & $\mathrm{Fp}$ & $\mathrm{Fp}$ & $\mathrm{Fp}$ & $\mathrm{Fp}$ & $\mathrm{Fp}$ & $\mathrm{Fp}$ & $\mathrm{Fp}$ & $\mathrm{Fp}$ & $\mathrm{Fp}$ & $\mathrm{Fp}$ \\
$\begin{array}{l}\text { Centrifugação/ } \\
\text { separação de } \\
\text { fases }\end{array}$ & $\mathrm{N}$ & $\mathrm{N}$ & $\mathrm{N}$ & $\mathrm{N}$ & $\mathrm{N}$ & $\mathrm{N}$ & $\mathrm{N}$ & $\mathrm{N}$ & $\mathrm{N}$ & $\mathrm{N}$ \\
\hline
\end{tabular}

Legenda: CH - Creme Homogêneo; F - Forte (formaldeído); E - Enxofre; S- Sem odor característico; Fp - formaldeído presente; Fa formaldeído ausente; $\mathrm{M}$ - Modificada (separação de fases); N - Normal (sem separação de fases).

Fonte: Dados da pesquisa (2018). 
Todas as amostras apresentaram teor de formaldeído no procedimento de doseamento por controle de identificação colorimétrico, sendo a identificação do formaldeído em uma concentração superior a $0,01 \%$ comprovada pelo surgimento de uma coloração rosa ou malva, apenas uma amostra informava a presença de formaldeído na composição.

Na presença dos fatos apresentados, alerta-se a importância da necessidade de maior vigilância sobre os produtos cosméticos comercializados e de campanhas educativas direcionadas aos cabeleireiros e consumidores sobre as implicações à saúde que o uso irregular de formaldeído em alisantes capilares pode provocar, bem como estimulá-los a comunicar às agências de vigilância sanitária os possíveis casos de irregularidades, na tentativa de coibir o uso inadequado do formaldeído, reduzindo assim os riscos de exposição (Crippa, Teixeira \& Rebello, 2015).

Em relação à centrifugação, todas as formulações mostraram-se sem separação de fases, precipitação, formação de caking ou coalescência.

Com o teste de centrifugação, determinou-se a instabilidade dos produtos emulsificados, já que a simulação de aumento da força de gravidade pode promover separação de componentes de diferentes densidades, como nas fases óleo e água.

$\mathrm{O} \mathrm{pH}$ dos fios de cabelo variam de 5,0 a 7.0. Formulações capilares com variações extremas de pH podem danificar o fio capilar, isso porque, o cabelo encolhe e enrijece ou até mesmo dissolve por completo em $\mathrm{pH}$ muito ácido ou aumenta a porosidade a medida que as camadas de cutícula se dilatam, obtendo uma aparência ressecada e opaca, chegando até dissolução completa do cabelo em pH fortemente alcalino, em concordância com isso a Anvisa em 2017, autorizou a utilização do ácido tioglicólico na concentração máxima de $11 \%(\mathrm{p} / \mathrm{p})$ profissionalmente e $8 \%(\mathrm{p} / \mathrm{p})$ para uso geral, apresentando valor de pH 9,5 e 7,0 respectivamente (Brasil, 2013). Os valores médios do pH encontrado nos alisantes em estudos foram identificados no Gráfico 1.

Gráfico 1: Valores médios do $\mathrm{pH}$ de alisantes capilares encontrados nos produtos.

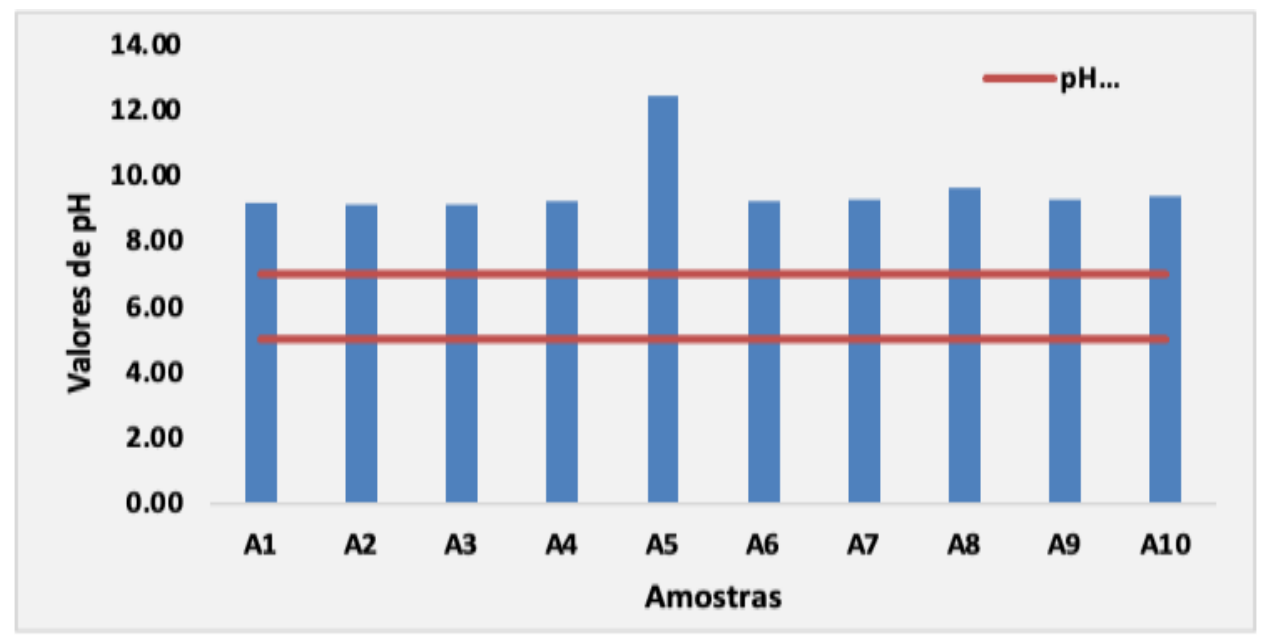

Legenda: Valores médios de $\mathrm{pH}$ das amostras A1-A10. Fonte: Dados da pesquisa (2018).

Diante do resultado obtido foi possível observar que das 10 amostras de alisantes capilares, todas elas (A1, A2, $\mathrm{A} 3, \mathrm{~A} 4, \mathrm{~A} 5, \mathrm{~A} 6, \mathrm{~A} 7, \mathrm{~A} 8, \mathrm{~A} 9$ e A10) ficaram fora do padrão exigido para que os alisantes sejam compatíveis com o pH fisiológico do couro cabeludo que é entre 5,0 a 7,0, com destaque para a amostra A5 (pH12) que ficou muito acima das demais (Brasil, 2012). 
Com a utilização de alisantes com pH acima do permitido pela ANVISA, reações adversas podem surgir e maior parte delas estão relacionadas à fragilidade da haste capilar e irritação no couro cabeludo, conforme o tempo de exposição e a quantidade utilizada do produto também podem provocar irritações no sistema respiratório e na pele, variando de leve a intensa (Sversur, 2017).

A viscosidade é uma medida da resistência de um sistema ao fluxo quando submetido a um estresse mecânico, quanto maior a viscosidade, maior será a resistência e a força a ser aplicada para produzir o fluxo com uma determinada velocidade, assim contribuindo ao auxílio no prognóstico dos efeitos da formulação e do processamento nas características do produto e na avaliação de sua qualidade e estabilidade (Brasil, 2019). Expressos seus valores de viscosidade das amostras no Gráfico 2.

Gráfico 2: Valores encontrados da viscosidade de cada amostra de alisante capilar expressos em centipoise (cP).

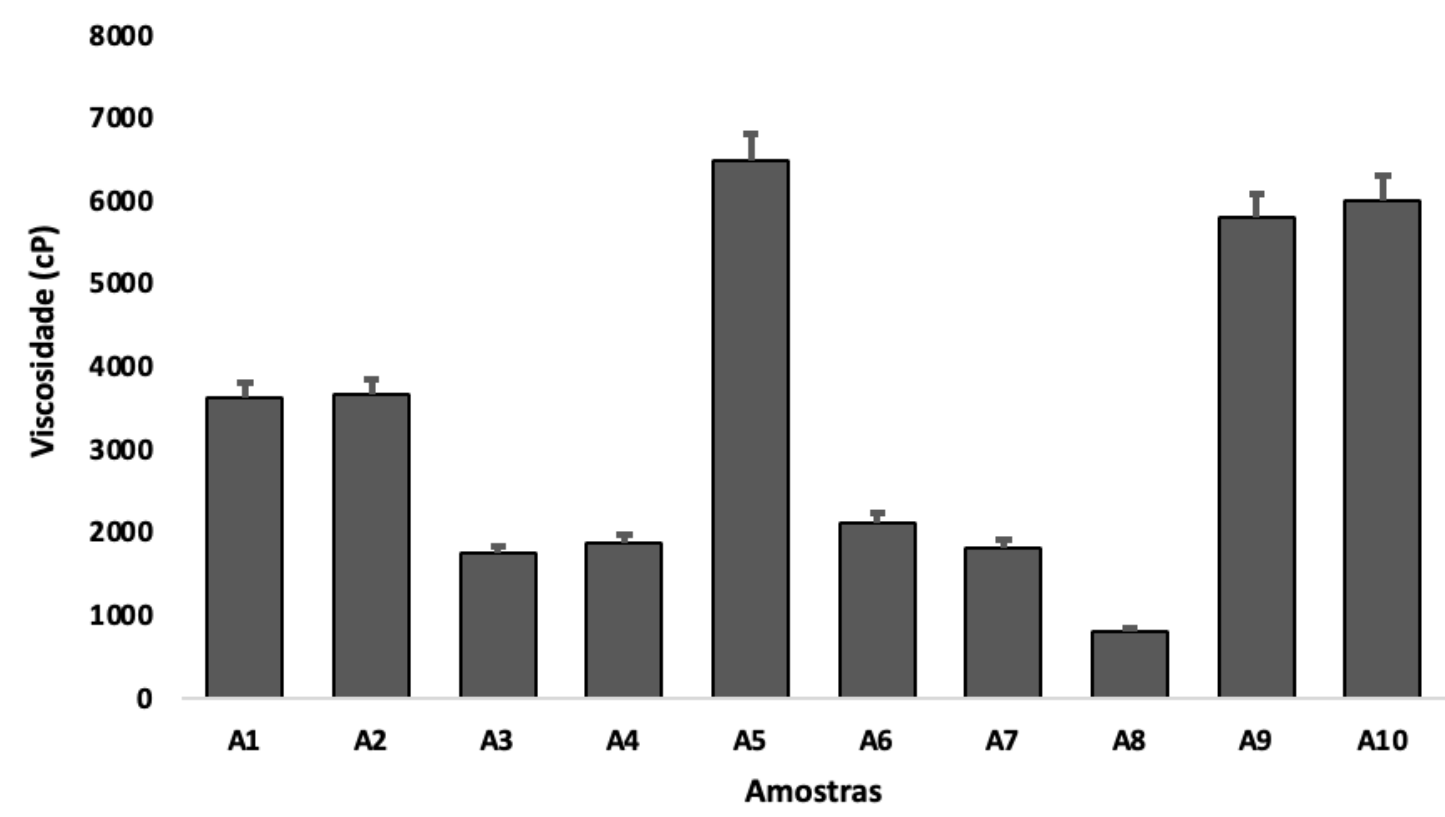

\footnotetext{
Legenda: Valores médios da viscosidade (cP) das amostras A1-A10. Fonte: Dados da pesquisa (2018).
}

O teste realizado para determinar a viscosidade apresentou maior valor para a amostra A5 (6486 cP), pois esta apresentou maior resistência do fluido ao fluxo. Por outro lado, a amostra com menor valor de viscosidade obtida foi a amostra A8 (801 cP).

Os valores de espalhabilidade obtidos para os alisantes capilares, em função do peso adicionado, estão representados no Gráfico 3 e revelam comportamentos paralelos para estas formulações, embora a amostra A8 tenha apresentado valores de espalhabilidade significativamente superior em relação as outras amostras. A amostra A10 obteve menos valor de espalhabilidade. 
Gráfico 3: Espalhabilidade das formulações em função do peso adicionado.

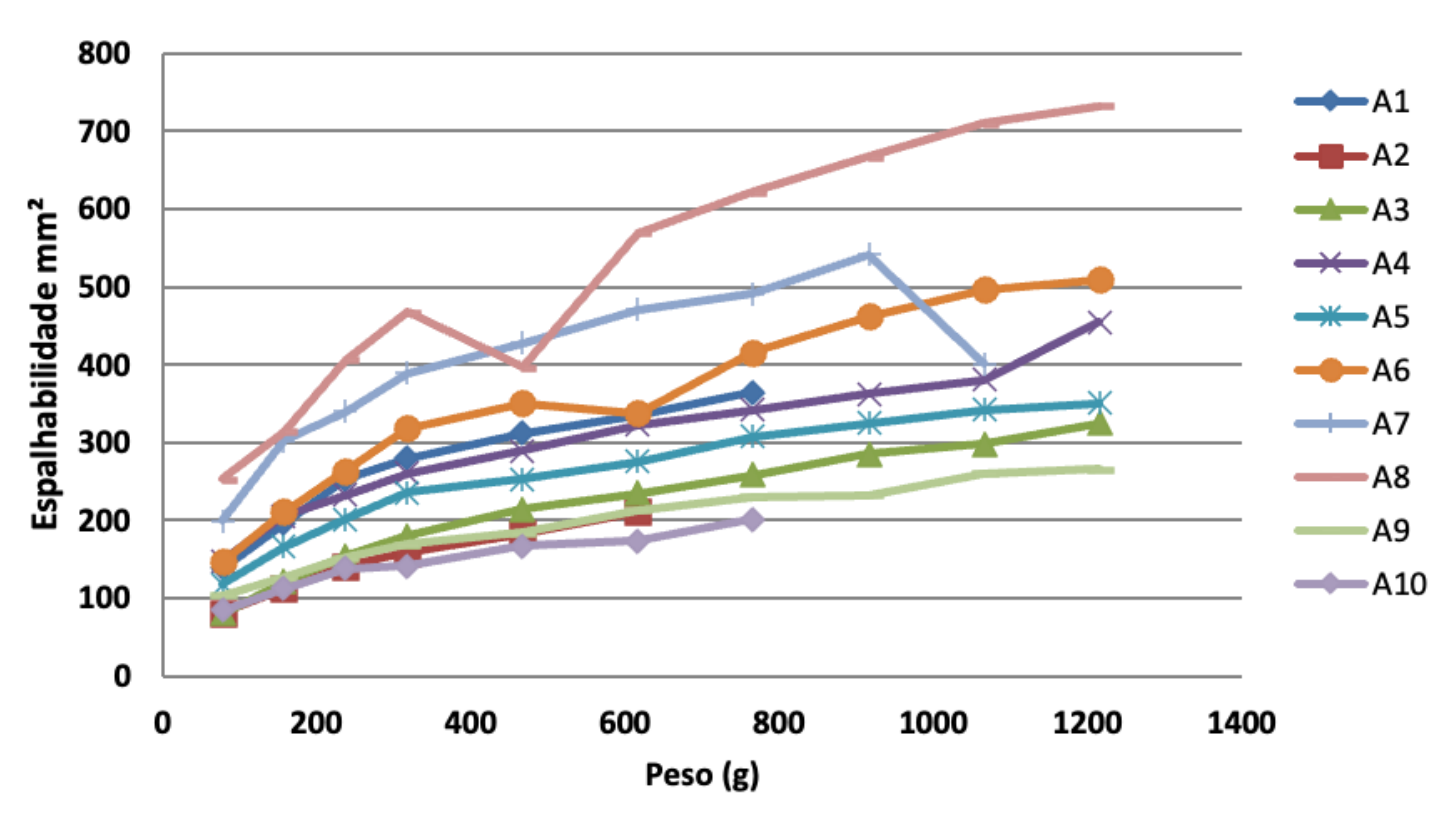

Fonte: Dados da pesquisa (2018).

O teste de espalhabilidade faculta determinar a capacidade que uma formulação apresenta de se espalhar quando for submetida a uma determinada força, procurando reproduzir as condições de esforço necessárias para aplicação na pele, dessa forma, os alisantes capilares devem propiciar fácil espalhamento sobre o couro cabeludo (Brasil, 2019).

Juntamente com composição do rótulo, existe a necessidade de uma maior vigilância acerca dos produtos cosméticos principalmente os alisantes capilares a base de formaldeído (substância capaz de provocar sinas e sintomas prejudiciais ao organismo humano), por isso, a importância da implantação do sistema de cosmetovigilância, para garantir a qualidade final dos produtos cosméticos, tendo em vista, principalmente a segurança, eficácia, e informação à ANVISA, ao fabricante e ao consumidor. Dessa forma, esse sistema poderá proporcionar inúmeros benefícios ao setor de cosméticos como um todo (Brasil, 2008). A verificação do rótulo e componentes permitidos foi possível ser verificados na Tabela 2.

Tabela 2: Análise dos rótulos dos produtos em estudo de acordo com os Anexos V e VI da RDC 07/2005.

\begin{tabular}{|c|c|c|c|c|c|c|c|c|c|c|}
\hline AMOSTRAS/ & & & & & & & & & & \\
\hline AVALIAÇÃO DO ROTULO & A1 & A2 & A3 & A4 & A5 & A6 & A7 & A8 & A9 & $\mathbf{A 1 0}$ \\
\hline Rotulagem & $S$ & $\mathrm{~S}$ & $S$ & $\mathrm{~S}$ & $S$ & $S$ & $S$ & $\mathrm{~S}$ & S & $\mathrm{S}$ \\
\hline Nome do produto & S & S & $\mathrm{S}$ & S & S & S & S & S & S & S \\
\hline Marca & S & S & $\mathrm{S}$ & S & S & S & S & S & S & S \\
\hline \multicolumn{11}{|l|}{ Número de registro } \\
\hline do produto & $S$ & $S$ & S & S & S & $S$ & $S$ & $\mathrm{~S}$ & $S$ & $\mathrm{~S}$ \\
\hline Lote ou Partida & S & S & S & S & S & S & S & S & S & $\mathrm{S}$ \\
\hline Prazo de Validade & S & S & S & S & S & S & S & S & S & S \\
\hline Conteúdo & S & S & S & S & S & S & S & S & S & $\mathrm{S}$ \\
\hline
\end{tabular}




\begin{tabular}{cccccccccccc}
\hline País de origem & $\mathrm{S}$ & $\mathrm{S}$ & $\mathrm{S}$ & $\mathrm{S}$ & $\mathrm{S}$ & $\mathrm{S}$ & $\mathrm{S}$ & $\mathrm{S}$ & $\mathrm{S}$ & $\mathrm{S}$ \\
Fabricante/Importador/Titular & $\mathrm{S}$ & $\mathrm{S}$ & $\mathrm{S}$ & $\mathrm{S}$ & $\mathrm{S}$ & $\mathrm{S}$ & $\mathrm{S}$ & $\mathrm{S}$ & $\mathrm{S}$ & $\mathrm{S}$
\end{tabular}

DomicílioFabricante/Importador/Titular

\begin{tabular}{|l|llllllllll} 
& $\mathrm{S}$ & $\mathrm{S}$ & $\mathrm{S}$ & $\mathrm{S}$ & $\mathrm{S}$ & $\mathrm{S}$ & $\mathrm{S}$ & $\mathrm{S}$ & $\mathrm{S}$ & $\mathrm{S}$ \\
Modo de Uso (se for o caso) & $\mathrm{S}$ & $\mathrm{S}$ & $\mathrm{S}$ & $\mathrm{S}$ & $\mathrm{S}$ & $\mathrm{S}$ & $\mathrm{S}$ & $\mathrm{S}$ & $\mathrm{S}$ & $\mathrm{S}$
\end{tabular}

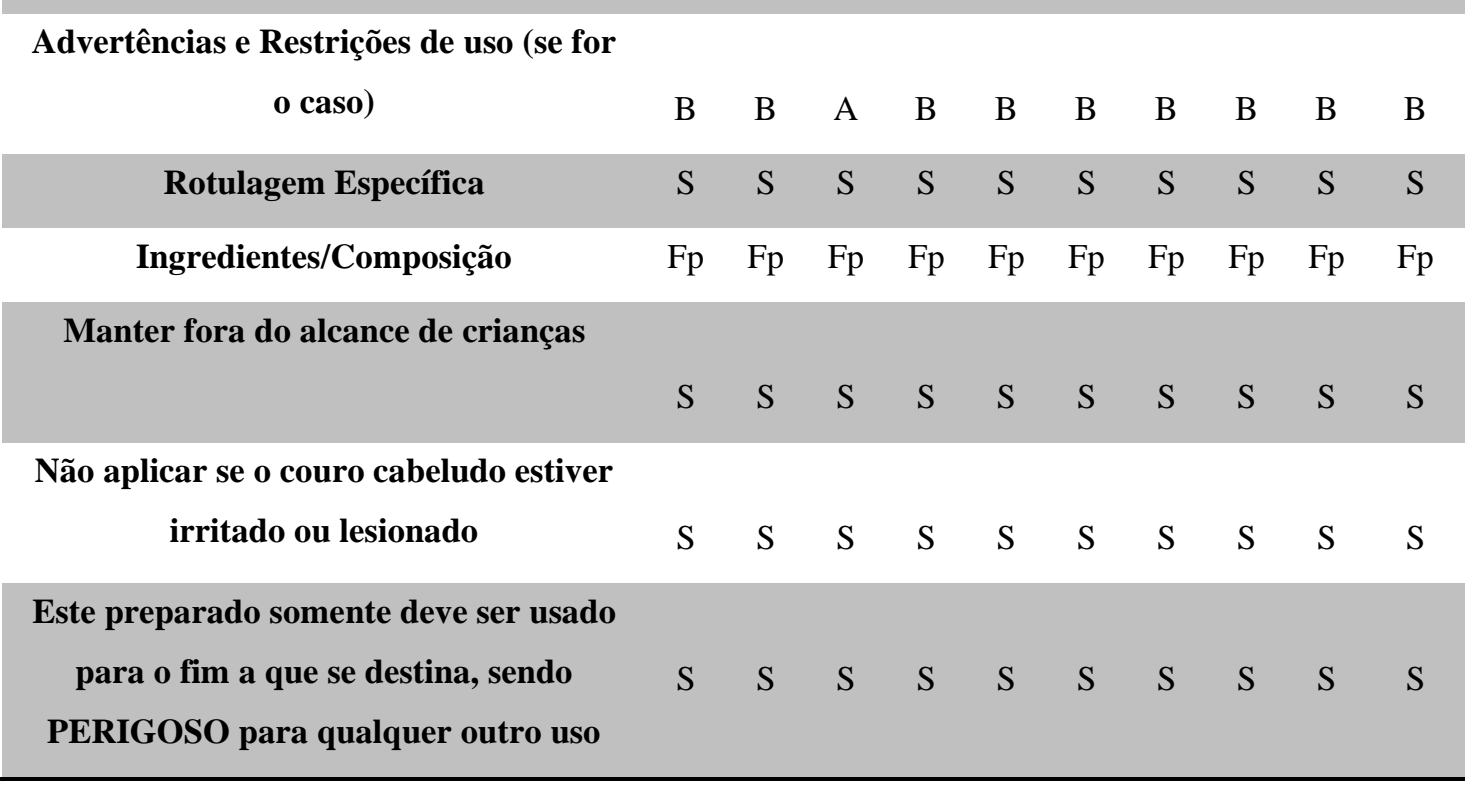

Legenda A- Concentração de formaldeído indicada; B - Concentração de formaldeído não indicada; N - Não indicado no rótulo; S Indicado no rótulo; $\mathrm{Fp}$ - formaldeído presente; $\mathrm{Fa}$ - formaldeído ausente.

Fonte: Dados da pesquisa (2018).

As amostras (A1, A2, A3, A4, A5, A6, A7, A8, A9 e A10) apresentaram teor de formaldeído no procedimento de doseamento por controle de identificação colorimétrico do formaldeído, no entanto, somente a amostra A3 indicava no rotulo a existência de formaldeído em sua composição como conservante, assim como exige a Anvisa. É válido ainda ressaltar que as amostras (A1, A2, A4, A5, A6, A7, A8, A9 e A10) não indicarem a presença da substância; todavia nenhuma apresentou irregularidades nos outros quesitos, juntamente com a amostra A3, seguindo os padrões exigidos pela RDC 07/2005. Conforme os dados obtidos em que todas as amostras foram positivas com a presença de formol, sabendo-se que ele pode vir apenas como conservante da amostra.

A aprendizagem contextual obtida pela leitura do rótulo de um produto permite obter aspectos relacionados à saúde quando este cumpre as regras que visam à compreensão ao usuário consumidor do produto, estando este ciente dos processos químicos de como agem essas substâncias para ser obtido o resultado esperado (Almeida, 2020).

\section{Conclusão}

O presente trabalho avaliou a qualidade dos alisantes capilares de acordo com as normativas sanitérias vigentes. Com isso, as análises de determinação do pH, características organolépticas, viscosidade, espalhabilidade, estabilidade da formulação por centrifugação e identificação colorimétrica permitiram observar a inadequação da maioria dos produtos avaliados frente a estes parâmetros.

Dessa maneira, existe a necessidade de uma maior vigilância acerca dos produtos cosméticos principalmente os alisantes capilares. Nesse sentido ressalta-se a importância da implantação do sistema de cosmetovigilância para garantir a qualidade final dos produtos cosméticos, que fortalece o cumprimento das exigências regulatórias, no que se refere à segurança 
e eficácia. Principalmente para alisantes capilares, em que é imprescindível a fiscalização quanto às adequações frente aos parâmetros para indústria.

O estudo presente abre lacunas para pesquisas posteriores acerca dos principais componentes que são necessárias maiores vigilância em sua adesão em alisantes capilares, como o formaldeído, por apresentarem alguns danos prejudiciais à saúde, quando utilizados indevidamente em produtos capilares.

\section{Referências}

ABIHPEC. (2017). Associação Brasileira da Indústria de Higiene Pessoal, Perfumaria e Cosméticos. Panorama do setor 2017. Acesso em junho de 2019.

Almeida Junior, E. do L.., Mueller, ER., \& Batalini, C.. (2020). Processos de endireitamento capilar como tema contextualizador para o ensino de Química Orgânica. Research, Society and Development , 9 (12), e12291210878. https://doi.org/10.33448/rsd-v9i12.10878

ANVISA. (2019). Agência Nacional de Vigilância Sanitária. Legislação específica da Área/Cosméticos.

ANVISA. (2019). Agência Nacional de Vigilância Sanitária. Regulamentação e Implantação da Cosmetovigilância no Brasil.

BRASIL. (2008). Ministério da Saúde. Agência Nacional de Vigilância Sanitária. Guia de Controle de Qualidade de Produtos Cosméticos. 2.ed. Brasília, DF: ANVISA, 121p.

BRASIL. (2021). Ministério da Saúde. Agência Nacional de Vigilância Sanitária. Lista de substâncias que os produtos de higiene pessoal, cosméticos e perfumes não devem conter. Resolução de Diretoria Colegiada (RDC) nº 151, de 11 de agosto de 2021.

BRASIL. (2020). Ministério da Saúde. Agência Nacional de Vigilância Sanitária. Procedimentos e requisitos para a regularização de produtos cosméticos para alisar ou ondular os cabelos. Resolução de Diretoria Colegiada (RDC) n 144, de 27 de junho de 2020.

BRASIL. (2012). Ministério da Saúde. Agência Nacional de Vigilância Sanitária. Resolução de Diretoria Colegiada (RDC) nº 3 , de 18 de janeiro de 2012.

BRASIL. (2015). Ministério da Saúde. Agência Nacional de Vigilância Sanitária. Resolução de Diretoria Colegiada (RDC) nº 7, de 10 de fevereiro de 2015.

BRASIL (2013). Ministério da Saúde. Agência Nacional de Vigilância Sanitária. Resolução de Diretoria Colegiada (RDC) nº 15 , de 26 de março de 2013.

BRASIL. (2005). Ministério da Saúde. Agência Nacional de Vigilância Sanitária. Resolução de Diretoria Colegiada (RDC) no 332 , de 01 de dezembro de 2005 .

BRASIL. (2019). Agência Nacional de Vigilância Sanitária (ANVISA). Cosmetovigilância.

BRASIL. (2008). Agência Nacional de Vigilância Sanitária. Guia de controle de qualidade de produtos cosméticos - uma abordagem sobre os ensaios físicos e químicos. $2^{\text {a }}$ edição, revista - Brasília: ANVISA.

BRASIL. (2000). Ministério da Saúde. Agência Nacional de Vigilância Sanitária. Resolução de Diretoria Colegiada (RDC) nº 79 , de 28 de agosto de 2000.

BRASIL. (2010). Ministério da Saúde. Agência Nacional de Vigilância Sanitária. Farmacopeia Brasileira (5² Edição). Brasília, DF, 546p.

BRASIL. (2019). Ministério da Saúde. Agência Nacional de Vigilância Sanitária. Farmacopeia Brasileira (6º Edição). Brasília, DF, 874p.

Crippa VO, Teixeira LRF, Rebello LC. (2015). Análise quali- quantitativa de formaldeído em amostras de produtos destinados ao alisamento capilar utilizados em salões de beleza no município de Linhares, ES-Brasil. Infarma. 27(1): 22-27.

Ferreira VT. (2015). Avaliação semi-quantitativa da concentração de formaldeído em formulações cosméticas de alisamento progressivo e selantes capilares. Ceilândia. 40 p. Monografia (Bacharelado em Farmácia), Universidade de Brasília Faculdade de Ceilândia. Ceilândia.

Knorst. (1991). Desenvolvimento tecnológico de forma farmacêutica plástica contendo extrato concentrado de Achyrocline satureioides Lam. Porto Alegre. 228 p. Dissertação de Mestrado. Universidade Federal do Rio Grande do Sul. Porto Alegre.

MERCOSUL. (2019). Mercado Comum do Sul MERCOSUL/GMC/res. 19/05 programas de cosmetovigilância na área de produtos de higiene pessoal, cosméticos, perfumes.

Vitola, J., Da Silva, G., \& de Oliveira, H. (2019). Avaliação do teor de formaldeído em amostras de alisantes capilares utilizados em salões de beleza de Pedro Gomes-MS, Brasil. Infarma - Ciências Farmacêuticas, 31(1), 28-33. 